\title{
Neurological aspects of sinoatrial heart block
}

\author{
A.J.FA I R F A X A N D C. D. L A M B E R T \\ From the Departments of Cardiology and Neurology, St George's Hospital, London
}

SYNOPSIS The symptoms of 100 patients with chronic cardiac sinoatrial disorder were analysed. The most common presenting features were syncope in 34 cases and dizziness in 22 cases. Over three-quarters of the patients had cereiral ischaemic symptoms at some stage of the disease. Diagnostic difficulties are often encountered and are illustrated by two case histories. Although sinoatrial disorder has been described in association with neuromuscular diseases, only one such example was found in this series. The patient had a limb girdle dystrophy with cardiomyopathy and diffuse disease of the cardiac conducting system. Muscle biopsy samples taken from 11 patients with idiopathic sinoatrial disorder were normal showing no evidence of subclinical muscular disease.

The importance of disorders of the sinoatrial pacemaker in producing disturbances of cardiac rhythm has become increasingly recognized by cardiologists in recent years (Moss and Davis, 1974). The resulting disturbances of rhythm have been collectively termed the 'sick sinus' syndrome. Patients with this disorder may present to a neurologist with recurrent episodes of altered consciousness, dizziness, or syncope. Diagnosis may be difficult unless cardiac monitoring facilities are available, especially when the routine electrocardiogram is normal. Embolism is an important complication occurring in $15-20 \%$ of cases and cerebral infarction is the most common outcome (Rubenstein et al., 1972; Fairfax, Lambert, and Leatham-submitted for publication 1976).

The majority of cases of the sick sinus syndrome are of unknown or of ischaemic aetiology. This syndrome occasionally develops in patients with neuromuscular diseases and has been reported in dystrophia myotonica (Radford and Julian, 1974), facioscapulohumeral dystrophy (Bloomfield and Sinclair-Smith, 1965; Caponnetto et al., 1968), scapuloperoneal dystrophy (Thomas et al., 1972), and Friedreich's ataxia (Théry, 1975). Sinoatrial disorder is also recognized in the Jervell and Lange-Nielsen syndrome in which congenital deafness is associated with syncope, a characteristic electrocardiogram, and sudden death from cardiac arrest (Schwartz et al., 1975).

Address for correspondence: Dr A. J. Fairfax, Department of Cardiology, St George's Hospital, Hyde Park Corner, London S.W.1.

(Accepted 22 January 1976.)
In this paper the presenting features, symptomatology, and frequency of neuromuscular associations of a group of patients with sinoatrial disorders are analysed. Diagnostic difficulties which may be encountered are illustrated by two case histories.

\section{METHODS}

One hundred patients attending cardiac clinics at St George's Hospital and the National Heart Hospital were studied (Table). All had documented, unexplained sinus bradycardia (heart rate less than 60 beats/minute) or sinoatrial arrest as a dominant feature. In some patients, this was associated with attacks of atrial tachycardia, flutter, and fibrillation (Rubenstein et al., 1972).

T A B L E

PRESENTING SYMPTOMS IN 100 PATIENTS WITH SINOATRIAL DISORDER

\begin{tabular}{lr}
\hline Symptom & (no.) \\
\hline Neurological & 34 \\
Syncope & 22 \\
Dizziness & 3 \\
Altered consciousness & \\
Cardiac & 12 \\
Palpitation & 11 \\
Chest pain & 5 \\
Shortness of breath & 2 \\
Heart failure & 11 \\
Incidental finding & \\
\hline
\end{tabular}


Details of the symptoms and of any possible aetiological factors were obtained from the case records. Fifty of the patients were examined personally by one of the authors (A. J. F.). In 11 patients with idiopathic sinoatrial disorder, a biopsy specimen was obtained from the deltoid muscle under local anaesthesia during the implantation of a cardiac pacemaker. The muscle sections were examined using conventional morphological and histochemical techniques (Dubowitz and Brooke, 1973).

\section{RESULTS}

The series included 51 men and 49 women with a mean age of 64.7 years at the time of study (range 17-93 years). The age of onset of the disorder is given in Fig. 1. In 75 patients no cause was found for the arrhythmia. Fifteen patients had symptoms suggesting the presence of ischaemic heart disease. In three cases, damage to the sinus node followed heart surgery. Two of the youngest patients, aged 17 and 20 years, were brothers. Although no other members of the family were apparently affected, it was thought that the disorder was hereditary (Spellberg, 1971).

In one patient, a 40 year old woman, sinoatrial disorder and atrioventricular heart block were features of cardiomyopathy associated with a limb-girdle muscular dystrophy which had become evident in childhood. Further details of this case are reported elsewhere (Lambert and Fairfax, 1976).

The presenting features are summarized in the Table. Syncope was the most common presenting feature occurring in 34 cases; in addition, a further 39 patients subsequently developed this symptom. Dizzy attacks were noted by 83 patients at some stage. The most common cardiac symptom was recurrent palpitation. In 11 cases a slow pulse had been found incidentally before any symptoms developed. To illustrate particular diagnostic difficulties, two case histories are described in detail.

\section{CASE 1}

A healthy 58 year old man presented as an emergency after a series of five blackouts on the morning of admission. Three months previously, episodes of light-headedness with facial pallor had developed; these became more severe, sometimes resulting in loss of consciousness. A similar blackout 15 years earlier had been regarded as epileptic and treatment with phenobarbitone instituted. There was no history of angina pectoris.

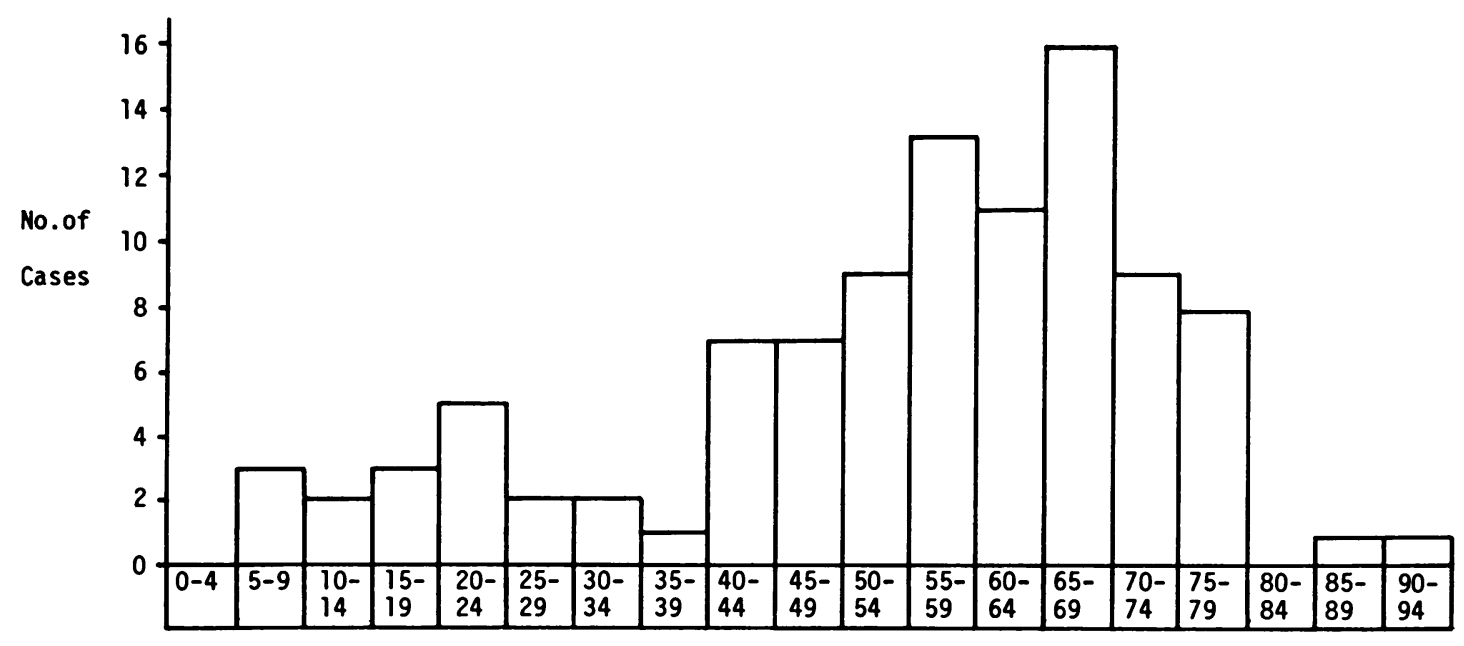

Age Group (years)

FIG. 1 Age at onset of symptoms of sinoatrial disorder. 
On examination, the patient was normotensive with no postural hypotension. There were no abnormal cardiological or neurological signs. The resting electrocardiogram was unremarkable apart from left axis deviation. Unlike many cases of sinoatrial disorder where constant sinus bradycardia is a feature (Fig. 2), the resting heart rate was 75 beats/minute, accelerating normally with exercise and infusions of isoprenaline and atropine, and slowing with carotid sinus massage.

An attack was documented in the cardiac monitoring unit: at the onset, there was progressive slowing of the heart to 37 beats/minute, followed by complete atrial and ventricular asystole producing transient loss of consciousness. Treatment by temporary endocardial pacing was instituted and a paced-beat counter showed that the heart rate was falling below 40 beats/minute between 10 to 15 times in a 24 hour period, mainly at night.

In view of a family history of ischaemic heart disease, coronary arteriography was performed. The angiogram showed a normally functioning left ventricle and there was no sign of cardiomyopathy or of coronary artery disease. A diagnosis of idiopathic sinoatrial disorder was made, and a permanent demand pacemaker installed, since which time the patient has been asymptomatic.

\section{CASE 2}

A 73 year old man was referred to the neurology clinic for the treatment of Parkinson's disease. In view of a long history of angina pectoris, he was not given L-dopa, but improved substantially on benzhexol and amantidine. Two years later he developed sudden shakiness during which his vision became purple. Some attacks were associated with a loss of consciousness but were not related to posture. Facial flushing was noticed at the end of an attack.

Examination disclosed tremor and rigidity of Parkinsonism affecting all limbs. Postural hypotension was present with a fall from $130 / 80 \mathrm{mmHg}$ lying to $80 / 60 \mathrm{mmHg}$ standing. The resting pulse was 80 beats/minute with intervals of missed beats. Of the several possible causes of syncope present, the cardiac arrhythmia was thought to be the dominant feature. There had been evidence of sinoatrial dysfunction secondary to ischaemic heart disease for the previous four years with episodes of sinus bradycardia and short periods of sinus arrest. Left bundle branch block was also shown on the electrocardiogram. The pulse rate was not speeded by either isoprenaline or atropine, and periods of sinus arrest of up to two seconds continued despite drug therapy. The symptoms were abolished by the implantation of a pacemaker.

RESULTS OF MUSCLE BIOPSY No evidence of significant muscle pathology was found in the 11 skeletal muscle samples obtained by biopsy from patients with idiopathic sinoatrial disorder including the two brothers with familial sinus node disease. Three biopsy specimens, all from elderly patients, showed minor changes, namely mild predominance of type 1 fibres in one case, changes of denervation in the second, and occasional atrophic fibres in the third.

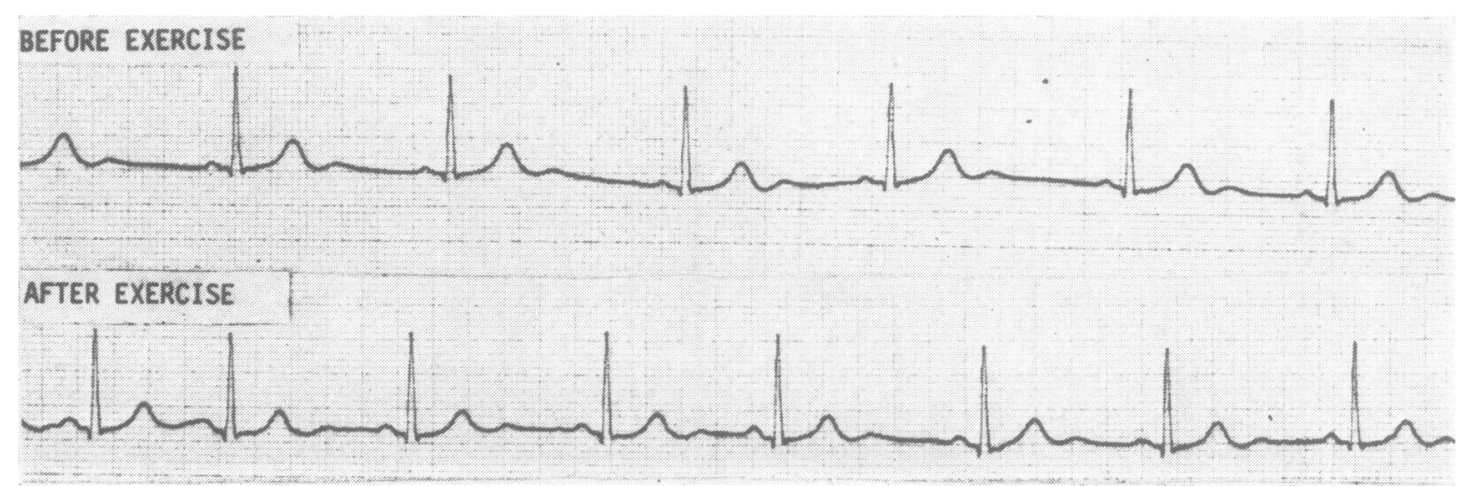

FIG. 2 Characteristic electrocardiogram of a case of sinoatrial disorder showing sinus bradycardia (rate 52/min) with little change of heart rate after maximal exercise. Time calibration $0.2 \mathrm{~s}$. 


\section{DISCUSSION}

The symptoms observed in sinoatrial disorder are predominantly episodes of disturbance or loss of consciousness, and are similar to those which occur in atrioventricular conduction defects. Both disorders predominantly affect patients in the sixth to eight decades of life, and both are now readily treatable by cardiac pacemaker implantation. The differential diagnosis in the elderly includes vertebrobasilar ischaemia, epilepsy, transient cerebral ischaemic attacks, and carotid sinus syncope.

Van Durme (1975) has provided evidence that many cases of cardiac rhythm disturbance are undetected by conventional means. $\mathrm{He}$ studied 95 patients who had presented with dizzy spells or syncope for which no cause was found on neurological or cardiological examination. The resting electrocardiogram showed a normal sinus rhythm in two-thirds of these patients. A portable, continuously recording electrocardiogram (Holter, 1961), however, showed that disturbances of cardiac rhythm, including unsuspected sinoatrial disorder, accounted for the symptoms of half of this group of patients. The normal electrocardiogram between attacks in some cases of sinoatrial disorder contrasts with that seen in intermittent atrioventricular heart block in which evidence of a peripheral conduction disturbance is usually present in the periods of sinus rhythm.

Although the hallmark of sinoatrial disorder is a sinus bradycardia refractory to drugs or exercise, case 1 illustrates that in the early stages of the disease bradycardia may be intermittent, and the heart may respond normally to chronotropic stimulation. Sinoatrial arrest may occur in isolation, or, characteristically, after a run of tachycardia (Fig. 3). Supra- ventricular tachycardia is a feature of sinus node dysfunction (but not of atrioventricular heart block) and this accounts for the frequency of palpitation in these patients. The alternation of slow and fast heart rates has given the disorder the alternative name of 'brady-tachy' syndrome (Short, 1954).

The majority of cases of sinoatrial disorder are of unknown aetiology (Pomerance and Davies, 1975), and there are few published postmortem studies. Ischaemic heart disease accounts for those cases in which the sinus node artery (an end-artery) is occluded during myocardial infarction (Rossi, 1962; Lippestad and Marton, 1967), but no vascular abnormality was found in a recent postmortem series (Brownlee et al., 1975). Davies and Pomerance (1972) have shown that the sinus node and atrial musculature undergo degenerative aging changes, and these may explain some cases of sinoatrial block in the elderly. Théry (1975) has pointed out that in his cases of sinoatrial block there was always complete destruction of the sinus node at necropsy. His series included one patient with Friedreich's ataxia and cardiomyopathy in whom the sinus node was destroyed by haemorrhage, fatty degeneration, and fibrosis. A high incidence of sinoatrial dysfunction has been reported in the cardiomyopathy of dystrophia myotonica (Perloff, 1973). The patient in our series with limb girdle muscular dystrophy also had evidence of a diffuse cardiomyopathy, with impairment of function both of the contractile myocardium and of the sinoatrial pacemaker and peripheral conducting tissues. It is evident from this study that neuromuscular disease accounts for a very small minority of patients with sinoatrial disorder, and that in the remainder of cases there is no evidence of skeletal muscle pathology.

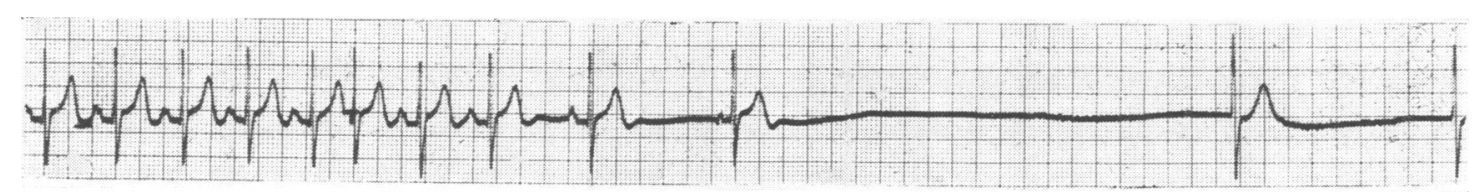

FIG. 3 Electrocardiogram recorded from a patient with the 'brady-tachy' syndrome, showing a run of supraventricular tachycardia followed by a period of cardiac asystole. Time calibration $0.2 \mathrm{~s}$. 
These patients, with a primary cardiac pathology, are still likely to have a neurological presentation with giddiness and syncope.

We wish to thank Dr A. Leatham and Dr P. GautierSmith for permission to study patients under their case, and for helpful criticism, and Miss $H$. Fischler for secretarial assistance. A.J.F. is in receipt of a grant generously provided from the St George's Hospital Research Fund.

\section{REFERENCES}

Bloomfield, D. A., and Sinclair-Smith, B. C. (1965). Persistent atrial standstill. American Journal of Medicine, 39, 335-340.

Brownlee, W. C., Evans, R. C., and Shaw, D. B. (1975). Pathology of conducting system in sinoatrial disease. British Heart Journal, 37, 779.

Caponnetto, S., Pastorini, C., and Tirelli, G. (1968). Persistent atrial standstill in a patient affected with facioscapulohumeral dystrophy. Cardiologia, 53, 341-350.

Davies, M. J., and Pomerance, A. (1972). Quantitative study of ageing changes in the human sinuatrial node and internodal tracts. British Heart Journal, 34, 150-152.

Dubowitz, V., and Brooke, M. H. (1973). Muscle Biopsy. A Modern Approach. Saunders: London.

Holter, N. J. (1961). New methods for heart studies. Science, 134, 1214-1220.

Lambert, C. D., and Fairfax, A. J. (1976). The neurological associations of chronic heart block. Journal of Neurology, Neurosurgery, and Psychiatry, 39, 571-575.
Lippestad, C. T., and Marton, P. F. (1967). Sinus arrest in proximal coronary artery occlusion. American Heart Journal, 74, 551-556.

Moss, A. J., and Davis, R. J. (1974). Brady-tachy syndrome. Progress in Cardiovascular Diseases, 16, 439-454.

Perloff, J. K. (1973). The myocardial disease of heredofamilial neuromyopathies. In Myocardial Diseases, pp. 319-335. Edited by N. O. Fowler. Grune and Stratton: New York.

Pomerance., A., and Davies, M. J. (1975). The Pathology of the Heart, p. 403. Blackwell: Oxford.

Radford, D. J., and Julian, D. G. (1974). Sick sinus syndrome: Experience of a cardiac pacemaker clinic. British Medical Journal, 3, 504-507.

Rossi, L. (1962). Infarction of the sinu-atrial node. British Medical Journal, 2, 927.

Rubenstein, J. J., Schulman, C. L., Yurchak, P. M., and De Sanctis, R. W. (1972). Clinical spectrum of the sick sinus syndrome. Circulation, 46, 5-13.

Schwartz, P. J., Periti, M., and Malliani, A. (1975). The long Q-T syndrome. American Heart Journal, 89, 378-390.

Short, D. S. (1954). The syndrome of altenating bradycardia and tachycardia. British Heart Journal, 16, 208-214.

Spellberg, R. D. (1971). Familial sinus node disease. Chest, 60, 246-251.

Théry, C. (1975). Pathology of sinoatrial disease. British Heart Foundation First European Symposium on Cardiac Arrhythmias (unpublished).

Thomas, P. K., Calne, D. B., and Elliott, C. F. (1972). X-linked scapuloperoneal syndrome. Journal of Neurology, Neurosurgery, and Psychiatry, 35, 208215.

Van Durme, J. P. (1975). Tachyarrhythmias and transient cerebral ischaemic attacks. American Heart Journal, 89, 538-540. 